\title{
Theory of lasing action in plasmonic crystals
}

\author{
J. Cuerda, F. Rüting, F. J. García-Vidal, ${ }^{*}$ and J. Bravo-Abad \\ Departamento de Física Teórica de la Materia Condensada and Condensed Matter Physics Center (IFIMAC), Universidad Autónoma de \\ Madrid, E-28049 Madrid, Spain
}

(Received 27 October 2014; published 26 January 2015)

\begin{abstract}
We theoretically investigate lasing action in plasmonic crystals incorporating optically pumped four-level gain media. By using detailed simulations based on a time-domain generalization of the finite-element method, we show that the excitation of dark plasmonic resonances (via the gain medium) enables accessing the optimal lasing characteristics of the considered class of systems. Moreover, our study reveals that, in general, arrays of nanowires feature lower lasing thresholds and larger slope efficiencies than those corresponding to periodic arrays of subwavelength apertures. These findings are of relevance for further engineering of active devices based on plasmonic crystals.
\end{abstract}

DOI: 10.1103/PhysRevB.91.041118

PACS number(s): 78.67.Pt, 73.20.Mf, 78.45.+h

Coherent light generation at the nanoscale is one of the critical stepping stones for the ultimate control of the light fields. In this context, plasmonic structures have recently emerged as versatile platforms for achieving lasing action at length scales well below the diffraction limit [1-16]. Very recently, it has been demonstrated that plasmon-assisted lasing action is not restricted to single nanocavity systems, but can be also observed in structures supporting extended plasmonic resonances, such as periodic arrays of metallic nanoparticles [17] and periodic arrays of subwavelength apertures milled in a metallic film [18]. These works reported independently on the unique ability of the corresponding metallic periodic structure (plasmonic crystal) to collect all the lasing light produced at the nanoscale and emit it to the far field in the form of a collimated beam. Although in a closely related context a detailed study of the spatiotemporal dynamics of lasing in gain-enhanced plasmonic nano-fishnet structures has been reported [19], a general study that explores the lasing properties of plasmonic crystals from a unified perspective is - to our knowledge-still lacking. In this work we address this issue and present a fundamental theoretical analysis of the dynamics and steady-state characteristics of lasing action in plasmonic crystals consisting of periodic arrays of metallic nanowires and subwavelength apertures embedded in an optically pumped four-level gain medium.

The insets of Figs. 1(a) and 1(b) render schematic views of the two model systems under study. For simplicity, we consider plasmonic crystals displaying one-dimensional periodicity along the $x$ direction and continuous translational symmetry along the $z$ direction (see the axes definition in the insets of Fig. 1). These structures support surface electromagnetic modes that resemble those decorating their two-dimensionally periodic counterparts [20] (this is particularly the case for thin-film plasmonic crystals, as the ones studied below). Therefore, the considered model systems are able to capture the fundamental physical phenomena governing the interaction of those surface modes with a four-level gain medium.

The first analyzed configuration [inset of Fig. 1(a)] consists of a one-dimensional periodic array $($ period $p)$ of rectangular

\footnotetext{
*fj.garcia@uam.es

†jorge.bravo@uam.es
}

gold nanowires of width $w$ and height $h$. The nanowire array is embedded in a dielectric host of index $n_{h}$ containing four-level organic dye molecules, which, when optically pumped, acts as a gain medium. This active medium extends a distance $t$ above and below structure. Lossless dielectric regions matching the host index extend infinitely above and below the active medium. The whole system is illuminated by a $p$-polarized plane wave (i.e., a plane wave with its $E$-field pointing along $x$ ) incident normally onto the structure. The second structure under study [inset of Fig. 1(b)] is similar to the above-described one, but in this case the nanowire array is replaced by a slit array defined by a periodicity $p^{\prime}$ and a height and a width of the metallic regions given by $h^{\prime}$ and $w^{\prime}$, respectively. The thickness of the active layers is $t^{\prime}$.

To compute the optical response of the considered active systems we use a numerical framework based on a time-dependent generalization of the finite element method (FEM-more details can be found in Refs. [21,22]). This $a b$-initio semiclassical approach is based on solving the field equation for the potential vector, $\mathbf{A}(\mathbf{r}, t), \nabla \times\left[\left(1 / \mu_{0}\right) \nabla \times\right.$ $\mathbf{A}]+\epsilon_{r} \epsilon_{0} \partial^{2} \mathbf{A} / \partial t^{2}=\partial \mathbf{P} / \partial t$, where $\mathbf{P}=\mathbf{P}(\mathbf{r}, t)$ is the timedependent part of the polarization (the time-independent contributions to the polarization are accounted for through the parameter $\left.\epsilon_{r}\right)$. Within the metallic regions of the system, $\mathbf{P}(\mathbf{r}, t)$ is computed through the conventional Drude-Lorentz form, taking the corresponding parameter values from a fit to available experimental data [23]. Inside the active medium, $\mathbf{P}(\mathbf{r}, t)$ has two different contributions, $\mathbf{P}(\mathbf{r}, t)=\mathbf{P}_{a}(\mathbf{r}, t)+\mathbf{P}_{e}(\mathbf{r}, t)$. These contributions arise from the stimulated absorption $\left[\mathbf{P}_{a}(\mathbf{r}, t)\right]$ and emission $\left[\mathbf{P}_{e}(\mathbf{r}, t)\right]$ of photons in the gain medium. In the particular case of a gain medium including four-level quantum emitters (with absorption and emission electronic transitions centered at $\omega_{a}$ and $\omega_{e}$, respectively), the dynamics of $\mathbf{P}_{i}(\mathbf{r}, t)(i=a, e)$ is governed by the following Lorentzian equation, $\partial^{2} \mathbf{P}_{i} / \partial t^{2}+2 \Gamma_{i} \partial \mathbf{P}_{i} / \partial t+\omega_{i}^{2} \mathbf{P}_{i}=-\sigma_{i} \Delta N_{i} \mathbf{E}_{i}$ with $\Gamma_{i}$ and $\sigma_{i}$ being the half linewidth and coupling strength of the corresponding transition. $\mathbf{E}_{i}=-\partial \mathbf{A}_{i} / \partial t$ is the local electric field. The functions $\Delta N_{i}=\Delta N_{i}(\mathbf{r}, t)$ represent the population inversion densities of the absorption and emission transitions, $\Delta N_{a}(\mathbf{r}, t)=N_{3}(\mathbf{r}, t)-N_{0}(\mathbf{r}, t)$ and $\Delta N_{e}(\mathbf{r}, t)=N_{2}(\mathbf{r}, t)-N_{1}(\mathbf{r}, t) . N_{j}(\mathbf{r}, t) \quad($ with $j=0, \ldots, 3)$ are the population densities of each of the energy levels of the quantum emitters. The temporal evolution of these 

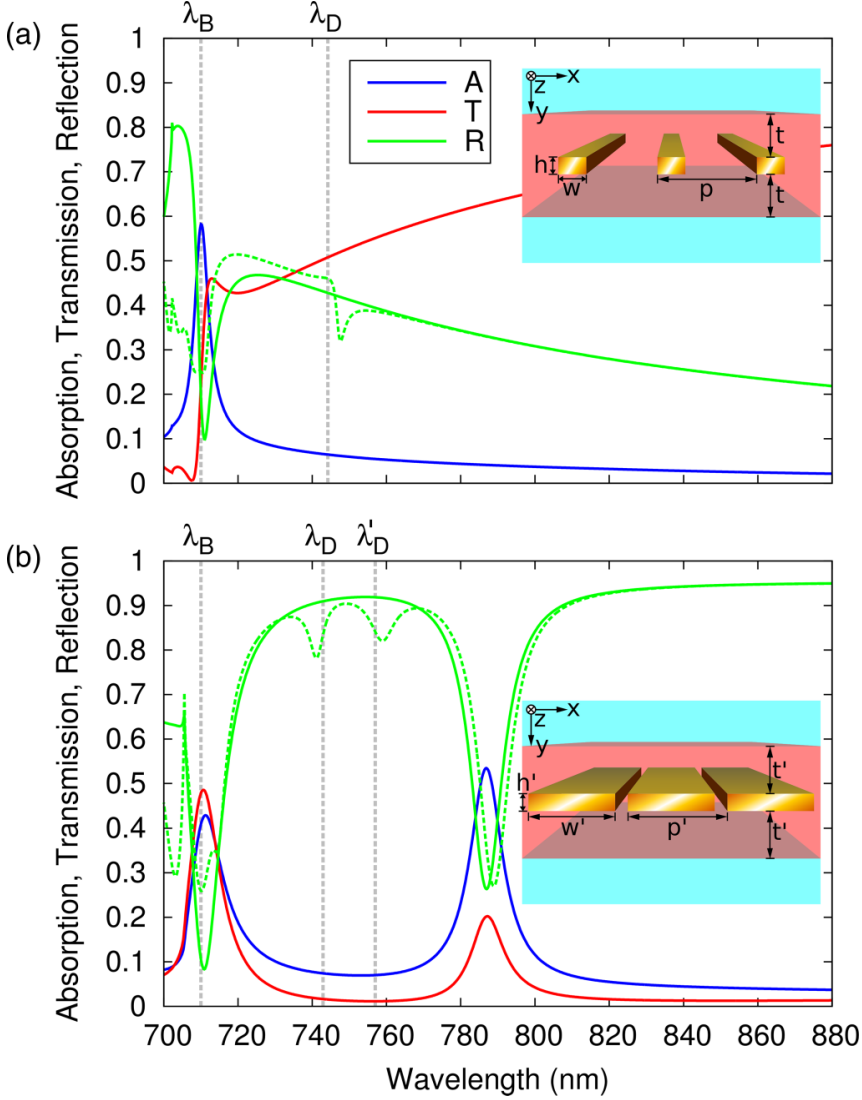

FIG. 1. (Color online) Passive spectral response of the studied array of nanowires (a) and slits (b). Solid blue, red, and green lines in both panels render, respectively, the computed absorption, transmission, and reflection obtained for normal incidence. The dashed vertical lines in each panel mark the location of the bright $\left(\lambda_{B}\right)$ and dark $\left(\lambda_{D}\right.$ and $\left.\lambda_{D}^{\prime}\right)$ plasmonic modes supported by each structure. Dashed green lines in both panels display the reflection spectra for an incident angle of $1^{\circ}$. The insets show schematic views of the considered systems, including the definition of the reference system and the geometrical parameters.

populations is, in turn, determined by the following rate equations:

$$
\begin{gathered}
\frac{\partial N_{3}}{\partial t}=-\frac{N_{3}}{\tau_{32}}+\frac{1}{2 \hbar \omega_{a}} \operatorname{Re}\left\{\frac{\partial \mathbf{P}_{a}}{\partial t} \mathbf{E}_{a}^{*}\right\}, \\
\frac{\partial N_{2}}{\partial t}=\frac{N_{3}}{\tau_{32}}-\frac{N_{2}}{\tau_{21}}+\frac{1}{2 \hbar \omega_{e}} \operatorname{Re}\left\{\frac{\partial \mathbf{P}_{e}}{\partial t} \mathbf{E}_{e}^{*}\right\}, \\
\frac{\partial N_{1}}{\partial t}=\frac{N_{2}}{\tau_{21}}-\frac{N_{1}}{\tau_{10}}-\frac{1}{2 \hbar \omega_{e}} \operatorname{Re}\left\{\frac{\partial \mathbf{P}_{e}}{\partial t} \mathbf{E}_{e}^{*}\right\}, \\
\frac{\partial N_{0}}{\partial t}=\frac{N_{1}}{\tau_{10}}-\frac{1}{2 \hbar \omega_{a}} \operatorname{Re}\left\{\frac{\partial \mathbf{P}_{a}}{\partial t} \mathbf{E}_{a}^{*}\right\},
\end{gathered}
$$

where $\tau_{j k}$ are the nonradiative decays between the $j$ th and $k$ th energy levels.

Thus, by solving the coupled set of nonlinear equations given by Eqs. (1)-(4), together with the field equation for $\mathbf{A}(\mathbf{r}, t)$ and the Lorentzian equation for $\mathbf{P}(\mathbf{r}, t)$, we obtain the whole spatiotemporal dynamics of the studied systems, including all the characteristics of their eventual laser emission. To that end, and in order to reduce the computational requirements of this problem, we introduce the following two additional steps. First, we exploit the fact that two characteristic frequencies of the system are known (namely, $\omega_{a}$ and $\left.\omega_{e}\right)$. This allows us to introduce the following ansatz on the functional form of the potential vector, $\mathbf{A}(\mathbf{r}, t)=$ $\mathbf{A}_{a}(\mathbf{r}, t) \exp \left(-i \omega_{a} t\right)+\mathbf{A}_{e}(\mathbf{r}, t) \exp \left(-i \omega_{e} t\right)$, where $\mathbf{A}_{i}(\mathbf{r}, t)$ are slowly varying complex amplitudes. We also assume that a similar expansion holds for $\mathbf{P}(\mathbf{r}, t)$. Note that the above ansatz for $\mathbf{A}(\mathbf{r}, t)$ does not impose any constraint on the spatial profile of the modes in the system or their harmonic oscillation frequencies [24]. In addition, this ansatz allows us to track separately the time evolution of the pump and emitted fields. Second, we rewrite Eqs. (1)-(4) in their so-called weak form [25]. By construction, the weak form makes the considered set of coupled nonlinear equations ideally suited to be solved with a FEM method [26]. This fact brings all the benefits of the adaptative meshing inherent to FEM methods to time-domain simulations of active plasmonic systems. On top of that, the application of periodic boundary conditions in this approach allows filtering the $k$-Bloch states that lie within the gain spectrum, and, thus, to reduce the significant complexity that characterizes many-mode gain competition phenomena.

Figures 1(a) and 1(b) (main panels) summarize the passive spectral response of the considered structures (i.e., the response obtained for a negligible density of organic molecules in the host dielectric). The geometrical parameters defining the nanowire array are $p=434 \mathrm{~nm}, w=122 \mathrm{~nm}, h=75 \mathrm{~nm}$, and $t=187 \mathrm{~nm}$, whereas those corresponding to the slit array are $p^{\prime}=436 \mathrm{~nm}, w^{\prime}=380 \mathrm{~nm}, h^{\prime}=75 \mathrm{~nm}$, and $t^{\prime}=208 \mathrm{~nm}$. To allow a meaningful comparison between both systems, we have chosen the same value of the metal thickness $h=h^{\prime}$, and a value of the ratio $t^{\prime} / t$ such that both systems feature the same amount of gain material. The rest of the geometrical parameters have been optimized to tune the resonance observed in the spectra at $\lambda \approx 710 \mathrm{~nm}$ to the stimulated emission from the gain medium described below. In both cases, the refractive index of the host medium is $n_{h}=1.62$. The above described structures have been also designed so that there are no transversal-electric-polarized modes within the absorption and emission linewidths. Therefore, we expect the polarization of the corresponding laser emission to be transversal-magnetic (i.e., laser light will be emitted with the same polarization of both the pump and the plasmonic resonances supported by the considered structures). Note that the three-dimensional counterparts of the class of systems analyzed in this work could feature polarization mixing effects that could, in turn, modify the laser light polarization with respect to that of the pump or the plasmonic resonances of the system.

By performing separate eigenmode calculations, we found that the resonance observed in the spectra of Figs. 1(a) and 1(b) at $\lambda \approx 710 \mathrm{~nm}$ corresponds, in both structures, to a mode of plasmonic nature (the computed wavelength of that eigenmode, $\lambda_{B}$, is marked by a vertical dashed line). In the case of the slit array, an additional resonant peak appears in the spectra at $\lambda \approx 790 \mathrm{~nm}$. This resonance can be ascribed to the excitation of a Fabry-Perot mode residing inside the slits [20]. Notably, our eigenmode analysis also reveals that both nanowire arrays and slit arrays support an additional class of plasmonic modes 
[located $\lambda_{D} \approx 744 \mathrm{~nm}$; see corresponding dashed lines in Figs. 1(a) and 1(b)], whose modal symmetry prevents them to be excited by normally incident radiation [27,28]. Figure 1 also illustrates the spectral response at non-normal incidence (for clarity, only the reflection spectra are displayed). As observed, even a slight deviation of $1^{\circ}$ from the normal incident condition leads to the excitation of the dark-mode resonances of the system. The results for the slit array also reveal that, in addition to the above-mentioned dark mode at $\lambda_{D}$, there exists another dark mode at $\lambda_{D}^{\prime} \approx 760 \mathrm{~nm}$. By examining the corresponding field profile, we found that this mode is the counterpart in our system of the short-range surface plasmon polariton arising in flat metallic films [29]. Due to the large ohmic losses featured by this mode, it does not play any significant role in the analysis of the lasing dynamics of the system. For definiteness, in the rest of this work we focus on the case of normal incidence.

We carried out a series of numerical experiments in the active counterparts of the structures described above. In these calculations, the emission wavelength of the fourlevel emitters, $\lambda_{e}$, was varied continuously from $\lambda_{e}=\lambda_{B}$ to $\lambda_{e}=\lambda_{D}$ (note that $\lambda_{e}=\lambda_{B}$ is the case considered in previous works $[17,18])$. We chose the following parameters for the emitters: $\lambda_{a}=680 \mathrm{~nm}, \tau_{21}=500 \mathrm{ps}, \tau_{32}=\tau_{10}=$ $100 \mathrm{fs}, \Gamma_{a}=\Gamma_{e}=1 /(20 \mathrm{fs}), \sigma_{a}=3.14 \times 10^{-16} \mathrm{~cm}^{2}$, and $\sigma_{e}=2.43 \times 10^{-16} \mathrm{~cm}^{2}$. These values model accurately the electronic properties of Rhodamine dye molecules [30]. We also chose a realistic value of the total density of molecules $N_{\text {tot }}=3 \times 10^{19} \mathrm{~cm}^{-3}$. Following Refs. [13,31], in order to account for the strong nonradiative quenching of the emitters located close to the metal surfaces, a gap of $10 \mathrm{~nm}$ in the active medium is assumed around the metallic regions of the studied systems. We have checked that varying the size of this gap does not have any significant influence on the fundamental findings reported in this work. Finally, the wavelength and amplitude of the external optical pump were fixed to $\lambda_{p}=\lambda_{a}$ and $\left|\mathbf{E}_{\text {pump }}\right|=0.75 E_{0}$, respectively ( $E_{0}$ is the so-called saturation electric field, a magnitude that only depends on the specific properties of the considered gain medium [32]).

Figure 2(a) renders the time evolution of the $\omega_{e}$ component of the $E$-field amplitude (spatially averaged over the computational domain), $\left\langle\left|\mathbf{E}_{e}(\mathbf{r}, t)\right|\right\rangle$, as computed for three representative values of $\lambda_{e}$. Only the calculations for the nanowire array are shown (similar results were obtained for the slit array). As expected for a large enough amplitude of the optical pump, all cases display the canonical signatures of lasing dynamics [33], characterized by sudden spikes of the signal that settle down to steady-state values for long times. In the analyzed configuration, the steady state has already been reached at $t \approx 350 \mathrm{ps}$ for all considered $\lambda_{e}$. These results show an unexpected nonmonotonic dependence with $\lambda_{e}$ of both the steady-state values of the field amplitude $\left\langle\left|\mathbf{E}_{e}\left(\mathbf{r}, t_{s d s t}\right)\right|\right\rangle$ and the lasing onset time $t_{0}$ (defined as the time at which the first lasing spike occurs). This nonmonotonic dependence is more clearly visualized in Fig. 2(b), where the results for $\left\langle\left|\mathbf{E}_{e}\left(\mathbf{r}, t_{s d s t}\right)\right|\right\rangle$ and $t_{0}$ calculated for a larger number of $\lambda_{e}$ values are displayed. Due to the presence of two plasmonic modes in the system, the steady-state field amplitude (the onset time) displays two local maxima (minima) appearing when $\lambda_{e}$ is tuned to $\lambda_{B}$ or $\lambda_{D}$ [see vertical dashed lines in Fig. 2(b)]. (a)

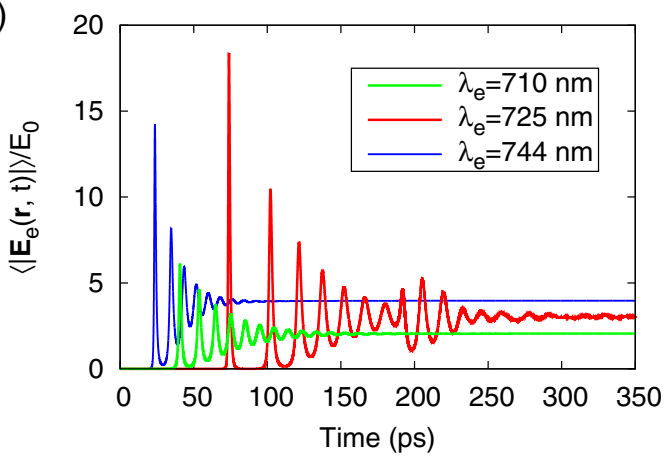

(b)

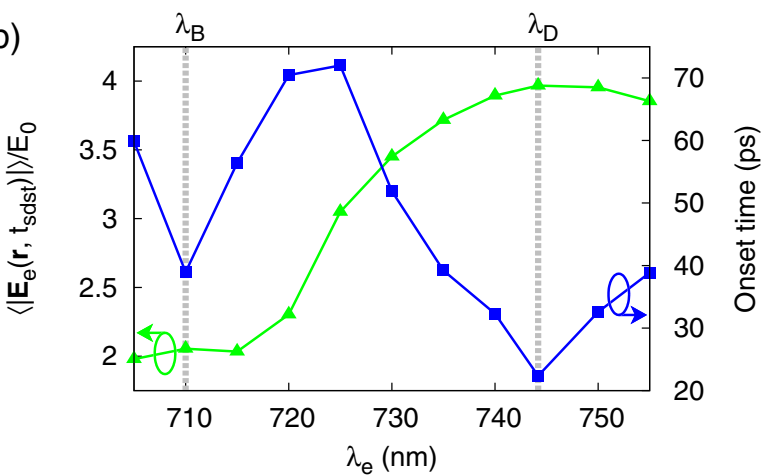

FIG. 2. (Color online) (a) Time evolution of the $\omega_{e}$ component of the calculated $E$-field (spatially averaged over the computational domain), $\left\langle\left|\mathbf{E}_{e}(\mathbf{r}, t)\right|\right\rangle$, for three different values of the emission wavelength: $\lambda_{e}=710 \mathrm{~nm}$ (green line), $\lambda_{e}=725 \mathrm{~nm}$ (red line), $\lambda_{e}=744 \mathrm{~nm}$ (blue line). The wavelength and amplitude of the pump field are, respectively, $\lambda_{p}=680 \mathrm{~nm}$ and $\left|\mathbf{E}_{\text {pump }}\right|=0.75 E_{0}\left(E_{0}\right.$ is the saturation electric field of the gain medium). The displayed results correspond to the nanowire array considered in Fig. 1(a). (b) Computed steady-state values for $\left\langle\left|\mathbf{E}_{e}(\mathbf{r}, t)\right|\right\rangle$ (green triangles, left $y$ axis) and lasing onset times (blue squares, right $y$ axis) as a function of the emission wavelength $\lambda_{e}$, as obtained for the same system as in (a).

Notably, the case $\lambda_{e}=\lambda_{D}$ displays a larger (smaller) value of $\left\langle\left|\mathbf{E}_{e}\left(\mathbf{r}, t_{s d s t}\right)\right|\right\rangle\left(t_{0}\right)$ than $\lambda_{e}=\lambda_{B}$. For intermediate values of $\lambda_{e}$, we observe how the mode competition between the bright and dark modes introduces (at $\lambda_{e} \approx 722 \mathrm{~nm}$ ) a qualitative change of the dependence of both $\left\langle\left|\mathbf{E}_{e}\left(\mathbf{r}, t_{s d s t}\right)\right|\right\rangle$ and $t_{0}$ on the emission wavelength. The signatures of this class of mode competition at the subwavelength scale are also manifested in time domain through a sudden change of the corresponding $E$ field amplitude oscillations [see the change of behavior of the red line in Fig. 1(a) at $t \approx 200 \mathrm{ps]}$. Similar mode competition phenomena at the subwavelength scale have been recently reported in Ref. [19] in the case of nanoplasmonic fishnet structures. The important point to realize is that a configuration in which the emission from the active medium is tuned to the main resonant feature of the spectrum (i.e., $\lambda_{e}=\lambda_{B}$ ) does not enable accessing the optimal lasing characteristics of this class of systems. As deduced from the above results, the optimal lasing regime is instead associated to the excitation (via the stimulated emission from the gain medium) of dark plasmonic resonances and, therefore, occurs when $\lambda_{e}=\lambda_{D}$. Much in the same way as occurs in the case of lasing action assisted by dark Fano modes in photonic crystals [34], laser photons can 


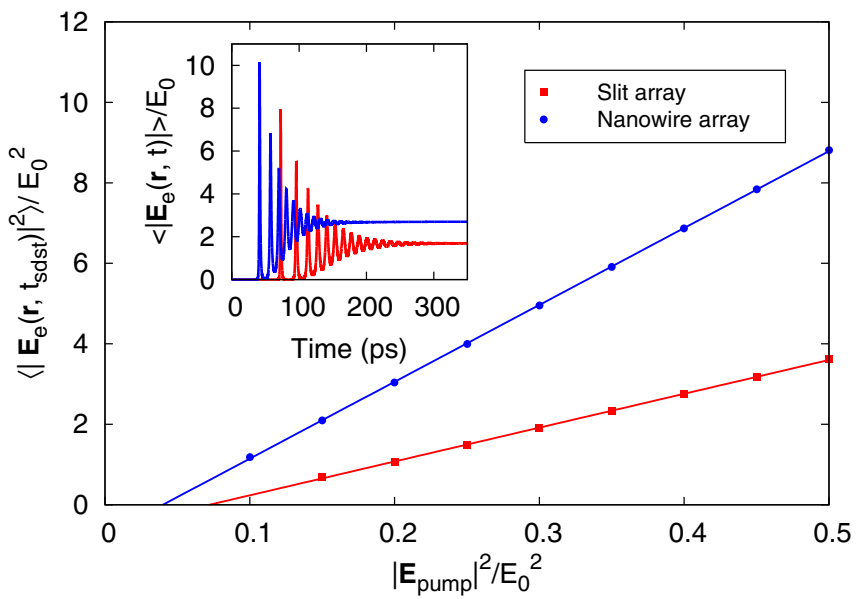

FIG. 3. (Color online) Computed steady-state values for $\left\langle\left|\mathbf{E}_{e}(\mathbf{r}, t)\right|^{2}\right\rangle$ as a function of $\left|\mathbf{E}_{\text {pump }}\right|^{2}$, as obtained for the nanowire array (blue circles) and the slit array (red squares). The geometrical parameters of both structures are optimized so that the corresponding dark plasmonic resonances are tuned to the emission wavelength $\lambda_{e}=710 \mathrm{~nm}$ of the gain medium. The inset shows the time evolution of the lasing field calculated with $\left|\mathbf{E}_{\text {pump }}\right|^{2}=0.5 E_{0}^{2}$.

be emitted from the sides of the system (i.e., emitted in the plane of periodicity), or, alternatively, in the vertical direction (normal to the plane of periodicity) by perturbing the perfectly periodic lattice of the considered plasmonic crystals.

Next, we compared the lasing characteristics of nanowire arrays and slit arrays operating at their corresponding optimal regimes. To do that, and in view of above conclusions, we fixed $\lambda_{e}=710 \mathrm{~nm}$ and optimized the geometrical parameters of both structures so that $\lambda_{e}$ is tuned to the dark plasmonic resonance supported by each structure. Specifically, in the rest of the Rapid Communication, we assume the set of parameters $t=200 \mathrm{~nm}, w=122 \mathrm{~nm}, h=75 \mathrm{~nm}$, and $p=410 \mathrm{~nm}$ for the nanowire array, and $t^{\prime}=224 \mathrm{~nm}, w^{\prime}=380 \mathrm{~nm}$, $h^{\prime}=75 \mathrm{~nm}$, and $p^{\prime}=410 \mathrm{~nm}$ for the slit array (note that, as in the previous case, the amount of gain material is the same in both structures). The rest of parameters, including the ones defining the gain medium, are the same as in the previous calculations. Figure 3 shows the corresponding steady-state values for $\left\langle\left|\mathbf{E}_{e}(\mathbf{r}, t)\right|^{2}\right\rangle$ as a function of $\left|\mathbf{E}_{\text {pump }}\right|^{2}$, as obtained for the nanowire array (blue circles) and the slit array (red squares). The linear dependence observed above the threshold corroborates that the two analyzed systems are indeed lasing. For illustration, the inset of Fig. 3 renders the time evolution of $\left\langle\left|\mathbf{E}_{e}(\mathbf{r}, t)\right|\right\rangle$ calculated for $\left|\mathbf{E}_{\text {pump }}\right|^{2}=0.5 E_{0}^{2}$. Importantly, as seen in Fig. 3, the nanowire array features a lower threshold and a significantly larger slope efficiency (by a factor larger than 2) than those obtained for the slit array. In this context, we point out that, as shown in Ref. [35], the inclusion of (amplified) spontaneous emission would result in the emergence of noise in the emitted laser signal. We do not, however, expect that those effects can change the above conclusion on the comparison of the slope efficiencies.

Finally, to obtain further insight into the physical origin of the above results, we apply to this problem a simple phenomenological model based on a spatially averaged formulation of the laser rate equations [31,36]. This model provides analytical expressions of the basic characteristics of a plasmonic laser in terms of a small set of effective parameters of the system. Specifically, within this approach, the expressions for the laser slope efficiency $\left(G_{e}\right)$ and the laser pump-rate threshold $\left(R_{\mathrm{th}}\right)$, derived from the dependence of the photon-number on the external pump rate, can be written as $G_{e}=Q / \omega_{e}$ and $R_{\mathrm{th}}=\omega_{e} S_{a} /\left(Q \Gamma v_{g} \eta \sigma_{e} \tau_{21}\right)$, respectively. Here $Q$ stands for the $Q$ factor of the considered modes, $S_{a}$ is the total area of the active media in the system, and $v_{g}$ is the group velocity of the plasmonic mode under analysis. The parameter $\Gamma$ is the so-called confinement factor that quantifies the degree of overlap between the mode profile and the active regions featured by the system, whereas $\eta$ provides a measure of the spatial-hole burning effects present in the lasing regime in the analyzed structures (detailed expressions for both magnitudes can be found in Refs. [31,36]).

For definiteness, in the following we focus on the application of this model to the numerical results shown in Fig. 3 (a completely similar analysis applies to the results of Fig. 2). To do that, we first computed the $Q$ factor of the dark mode supported by the nanowire and slit arrays considered in Fig. 3, and obtained $Q=215$ and $Q=130$, respectively. Then, using the simple expression for $G_{e}$ provided above, we found that the applied model predicts slope efficiencies of $G_{e}=8.1 \times 10^{-14} \mathrm{~s}$ and $G_{e}=4.9 \times 10^{-14} \mathrm{~s}$ for the nanowire array and slit array lasers, respectively. For comparison, the full numerics yields $G_{e}=9.2 \times 10^{-14}$ s for the nanowire array, and $G_{e}=4.8 \times 10^{-14}$ s for the slit array. The good quantitative agreement between model and full numerics demonstrates that the observed larger slope efficiency of the nanowire array can be exclusively ascribed to the larger $Q$ factor of its dark mode.

Next, using the steady-state field and population inversion distributions obtained from our full laser simulations, we computed the three parameters $\Gamma$, $\eta$, and $v_{g}$, entering the analytical expression for $R_{\text {th }}$ given above. From those values, together with the corresponding $Q$ factors and active material parameters, we obtained $R_{\mathrm{th}}=2.1 \times 10^{21} \mathrm{~s}^{-1} \mathrm{~m}^{-1}$ for the nanowire array and $R_{\mathrm{th}}=3.0 \times 10^{21} \mathrm{~s}^{-1} \mathrm{~m}^{-1}$ for the slit array. Importantly, in both structures we numerically found similar values of $v_{g}, \Gamma$, and $\eta$. This suggests that the physical origin of the smaller lasing threshold displayed by the nanowire array can be also ascribed to the larger $Q$ factor featured by the dark mode decorating that system. We note here that the model results for $R_{\text {th }}$ show only qualitative agreement with the full numerics, which predicts $R_{\mathrm{th}}=1.14 \times 10^{21} \mathrm{~s}^{-1} \mathrm{~m}^{-1}$ and $R_{\mathrm{th}}=1.48 \times 10^{21} \mathrm{~s}^{-1} \mathrm{~m}^{-1}$ for the nanowire array and the slit array, respectively. This points to the fact that, although in this class of systems a phenomenological model can be used to unveil qualitatively the physical origin of the observed lasing behavior, a full ab-initio nonlinear approach is needed to obtain accurate predictions of all lasing characteristics.

In conclusion, we have presented a unified theoretical study of lasing action in plasmonic crystals. We have found that dark modes in plasmonic crystals enable accessing the optimal lasing characteristics of this class of systems. In addition, by comparing lasing action assisted by dark modes in both nanowire arrays and slit arrays, we have shown that nanowire arrays feature smaller thresholds and larger lasing slope efficiencies that those corresponding to slit arrays. These results could be of importance for further development of novel large-area light-emitting structures based on active plasmonic crystals. 
[1] D. J. Bergman and M. I. Stockman, Phys. Rev. Lett. 90, 027402 (2003).

[2] M. T. Hill, Y.-S. Oei, B. Smalbrugge, Y. Zhu, T. de Vries, P. J. van Veldhoven, F. W. M. van Otten, T. J. Eijkemans, J. P. Turkiewicz, H. de Waardt, E. J. Geluk, S.-H. Kwon, Y.-H. Lee, R. Notzel, and M. K. Smit, Nat. Photon. 1, 589 (2007).

[3] M. Noginov, G. Zhu, A. M. Belgrave, R. Bakker, V. M. Shalaev, E. E. Narimanov, S. Stout, E. Herz, T. Suteewong, and U. Wiesner, Nature (London) 460, 1110 (2009).

[4] R. F. Oulton, V. J. Sorger, T. Zentgraf, R.-M. Ma, C. Gladden, L. Dai, G. Bartal, and X. Zhang, Nature (London) 461, 629 (2009).

[5] R.-M. Ma, R. F. Oulton, V. J. Sorger, G. Bartal, and X. Zhang, Nat. Mater. 10, 110 (2011).

[6] Y.-J. Lu, J. Kim, H.-Y. Chen, C. Wu, N. Dabidian, C. E. Sanders, C.-Y. Wang, M.-Y. Lu, B.-H. Li, X. Qiu, W.-H. Chang, L.-J. Chen, G. Shvets, C.-K. Shih, and S. Gwo, Science 337, 450 (2012).

[7] J. Seidel, S. Grafström, and L. Eng, Phys. Rev. Lett. 94, 177401 (2005).

[8] M. Ambati, S. H. Nam, E. Ulin-Avila, D. A. Genov, G. Bartal, and X. Zhang, Nano Lett. 8, 3998 (2008).

[9] M. A. Noginov, G. Zhu, M. Mayy, B. A. Ritzo, N. Noginova, and V. A. Podolskiy, Phys. Rev. Lett. 101, 226806 (2008).

[10] I. De Leon and P. Berini, Nat. Photon. 4, 382 (2010).

[11] S. Xiao, V. P. Drachev, A. V. Kildishev, X. Ni, U. K. Chettiar, H.-K. Yuan, and V. M. Shalaev, Nature (London) 466, 735 (2010).

[12] S. Wuestner, A. Pusch, K. L. Tsakmakidis, J. M. Hamm, and O. Hess, Phys. Rev. Lett. 105, 127401 (2010).

[13] J. M. Hamm, S. Wuestner, K. L. Tsakmakidis, and O. Hess, Phys. Rev. Lett. 107, 167405 (2011).

[14] O. Hess, J. B. Pendry, S. A. Maier, R. F. Oulton, J. M. Hamm, and K. L. Tsakmakidis, Nat. Mater. 11, 573 (2012).

[15] P. Berini and I. De Leon, Nat. Photon. 6, 16 (2012).

[16] R.-M. Ma, R. F. Oulton, V. J. Sorger, and X. Zhang, Laser Photon. Rev. 7, 1 (2013).

[17] W. Zhou, M. Dridi, J. Y. Suh, C. H. Kim, D. T. Co, M. R. Wasielewski, G. C. Schatz, and T. W. Odom, Nat. Nanotechnol. 8, 506 (2013).
[18] F. van Beijnum, P. J. van Veldhoven, E. J. Geluk, M. J. A. de Dood, G. W. 't Hooft, and M. P. van Exter, Phys. Rev. Lett. 110, 206802 (2013).

[19] S. Wuestner, J. M. Hamm, A. Pusch, F. Renn, K. L. Tsakmakidis, and O. Hess, Phys. Rev. B 85, 201406(R) (2012).

[20] F. J. Garcia-Vidal, L. Martin-Moreno, T. W. Ebbesen, and L. Kuipers, Rev. Mod. Phys. 82, 729 (2010).

[21] C. Fietz and C. M. Soukoulis, Opt. Express 20, 11548 (2012).

[22] F. Rüting, J. Cuerda, J. Bravo-Abad, and F. J. Garcia-Vidal, Laser Photon. Rev. 8, L65 (2014).

[23] P. B. Johnson and R. W. Christy, Phys. Rev. B 6, 4370 (1972).

[24] Frequency-pulling effects are included in our formalism through an additional harmonic time dependence in the slowly varying amplitudes $\mathbf{A}_{a, e}(\mathbf{r}, t)$, i.e., $\mathbf{A}_{a, e}(\mathbf{r}, t)=\tilde{\mathbf{A}}_{a, e}(\mathbf{r}, t) \exp \left(i \Delta \omega_{a, e} t\right)$, with $\Delta \omega_{a, e}$ being the frequency shift with respect to the corresponding carrier frequency $\omega_{a, e}$.

[25] J. Jin, The Finite Element Method in Electromagnetics (Wiley, New York, 2002).

[26] We use the FEM weak-form module provided within the commercial package Comsol Multiphysics (Comsol, Inc).

[27] B. Auguié and W. L. Barnes, Phys. Rev. Lett. 101, 143902 (2008).

[28] G. Vecchi, V. Giannini, and J. Gómez Rivas, Phys. Rev. B 80 , 201401 (2009).

[29] P. Berini, Adv. Opt. Photon. 1, 484 (2009).

[30] P. Sperber, W. Spangler, B. Meier, and A. Penzkofer, Opt. Quantum Electron. 20, 395 (1988).

[31] T. Pickering, J. M. Hamm, A. F. Page, S. Wuestner, and O. Hess, Nat. Commun. 5, 4972 (2014).

[32] R. Marani, A. D’Orazio, V. Petruzzelli, S. G. Rodrigo, L. MartinMoreno, F. Garcia-Vidal, and J. Bravo-Abad, New J. Phys. 14, 013020 (2012).

[33] A. E. Siegman, Lasers (University Science Books, Mill Valley, 1986).

[34] S. L. Chua, Y. Chong, A. D. Stone, M. Soljacic, and J. BravoAbad, Opt. Express 19, 1539 (2011).

[35] A. Pusch, S. Wuestner, J. M. Hamm, K. L. Tsakmakidis, and O. Hess, ACS Nano 6, 2420 (2012).

[36] S.-W. Chang and S. L. Chuang, IEEE J. Quantum Electron. 45, 1014 (2009). 